\title{
THE DRONES ARE COMING. WHAT TO CHOOSE? LOW AND MEDIUM ALTITUDE AERIAL ARCHAEOLOGY ON LIMES TRANSALUTANUS.
}

\begin{abstract}
Aerial archaeology has more than one century of tradition as a valuable research method. Like archaeology, the aerial reconnaissance is undergoing dynamic changes. The field must face the profound conceptual challenges raised by the evolving demands of archaeologists. In addition, aerial archaeology has to adapt its own methods in order to constantly incorporate new technologies such as: thermal vision, LiDAR and also advanced photogrammetry processing techniques. One of the greatest challenges and promising perspectives for evolution of the field is the arriving and rapidly spreading of small remote controlled aerial vehicles (UAVs - Unmanned Aerial Vehicles, known also as drones). However, a real development of the UAVs based aerial archaeology's branch is conditioned by the availability of special tailored aerial vehicles for archaeologist's needs. Unfortunately, the time has not yet come for this, while the major efforts in drones development is spent for aerial videography applications, surveillance and general entertainment. The implementation of a research project, dedicated to the longest built sector of the Roman limes in Dacia - Limes Transalutanus, represented for the authors a suitable occasion to assess the possibilities and limits of the large scale aerial archaeology based on UAVs. On the occasion there were tested two custom flying platforms and one commercial, multiple flight strategies and several processing algorithms. The linear nature and the extent of the site (basically a corridor of $157 \mathrm{~km}$ in length) called for distinct augmentation of equipment and survey workflows, with applicability in 'corridor' archaeological projects like those for highways and utilities networks.
\end{abstract}

Keywords: UAV, SfM, aerial archaeology, photogrammetry, roman limes

\section{DEMOCRATIZATION OF AERIAL ARCHAEOLOGY}

s the European Aviation Safety Agency (EASA) acknowledged recently ${ }^{1}, 2013$ was considered the year of drones ${ }^{2}$. The technology is nne of the fastest growing on a global market ${ }^{3}$ and several application

\section{Dan Ștefan}

Vector Studio Bucharest danstefan00@gmail.com

\section{Maria-Magdalena Ștefan}

Institute of Archaeology 'Vasile Pârvan' Bucharest m_magdalena.stefan@yahoo.com

\footnotetext{
Concept for Operations of Drones, 2015, 2.

2 The terms drones or UAVs (Unmanned Aerial Vehicles) designate either aircrafts flying without pilot/ UAS (Unmanned Aircraft Systems) or flying systems with remote control/RPAS (Remotely piloted aircraft systems) which can be considered a sub-category of the first. Such a flying system contains the entire ensemble of a rigid frame, propulsion system, flight controller, navigation system/navi-controller, power supply modules, telemetry and communication subsystem, dampling vibration platform for photo-video camera or other types of air-carried sensors.

3 Industry which reached by 2014 a global value greater than 660 million dollars, estimated that by 2020 will reach 5.59 billion dollars according to Report SE 3099 October 2015.
} 
fields (agriculture, geodesy, entertainment) have already experienced considerable change in their general data acquisition workflows ${ }^{4}$. Archaeology is one of these domains where drones have been making a difference by opening the regular archaeologists' access to aerial survey ${ }^{5}$, a previously highly specialized and costly investigation method based on man-piloted aircrafts ${ }^{6}$. Reasons for this significant evolution are both technical developments (like the advances of lithium-ion polymer batteries for discharging electrical power at very high intensity, the miniaturization of sensors, including gyroscopes, GPS, telemetry) but also political, as general tendencies advocate for relaxation of rules in the civilian field of applications ${ }^{7}$.

It is, however, the latest development in computer graphics methods of generating virtual reality based on photogrammetric algorithms ${ }^{8}$ that has been transforming drones into such a powerful tool for archaeologists by offering low-cost and easy to use means to obtain digital models of the terrain and archaeological landscapes from aerial images recorded with UAVs, without prerequisites as specially designed cameras or employment of complicated optical calibrations procedures ${ }^{9}$.

Drones as easy-to-use in archaeology remains, though, a rather fluid concept and amidst the general enthusiasm, we shouldn't ignore the fact that this technological immediateness has, at least for the moment, a major drawback - the lack of standardized data acquisition procedures and inconsistency between the experimental nature of archaeological research and the development rhythm of commercial products.

The implementation of a research project ${ }^{10}$, dedicated to the longest built sector of the Roman limes in Dacia Limes Transalutanus, represented for the authors a suitable occasion to assess the possibilities and limits of the aforementioned technologies. Putting into practice the UAV reconnaissance module proved to be a significant challenge,

\footnotetext{
4 Report SE 3099 October 2015

FERNÁNDEZ-HERNANDEZ et al. 2015, FERNÁNDEZ-LOZANO/ GUTIÉRREZ-ALONSO 2016; MOZAS-CALVACHE et al. 2012. Ongoing projects are listed in the dedicated international conferences: TOPOI Berlin 2014 (https://community.topoi.org/web/uav-2014/programm); From Aerostats to Drones: aerial imagery in Archaeology, Rome, Feb. 2016, Univ. Salento, Univ. Ghent, Univ. Cassino (http://www.archeologia-aerea.it)

6 Some recent reviews on aerial archaeology in Romania in OLTEAN 2007; PALMER/OBERLANDER/BEM 2009; BERECKI/ CZAJILIK 2012; RUS 2015.

Flight control regulations currently in use in Europe consider drones weighting over $150 \mathrm{~kg}$ as aircrafts, while the lighter ones are left to particular treatment according to national legislation. A law project aiming to unify security regulation at European level was proposed for analysis to the European Commission in September 2015. This text argues against weight criterion, focussing instead on how and in what conditions drones are being used (for example assessing the risk level for third parties). It does not deal however with personal privacy and data protection. In Romania, during January 2014 - January 2016, there have been enforced strict regulation on drones use in the civil sector (by Ministry Order). Presently, the legal environment is more favourable, still it remains incompletely defined.

8 For example: SIFT - Scale Invariant Feature Transform TRIGGS et al. 2000; bundle adjustment ZHANG et al. 2012; MVS - Multi-View Stereo BERGER et al. 2014; FURUKAWA/PONCE 2010; REMONDINO et al. 2014.

9 About calibration in REMONDINO/FRASER 2006

10 Romanian PNCDII funded project in the Partnerships Programme 'Interdisciplinary technology for investigating the archaeological heritage. Study case: the southern sector of Limes Transalutanus' http://limes-transalutanus.
} ro/\#en (TEODOR/ȘTEFAN M. 2014; TEODOR/STEFAN D. 2014) considering that the essential technical components of the flying platform, as required to cover the spatial extent of our targeted site (a corridor of $157 \mathrm{~km}$ in length and at least 400 m in width), had not been available for commercial purchase. Consequently, the team decided to design, build and test custom flying systems without pilot on board. Experimenting was also done in the methodological direction by comparing the results of various flying and data acquisition strategies, processing algorithms and georeferencing methods. Final comparison with commercial UAVs was also performed, as with aerial surveys' performance of piloted aircrafts on similar targets ${ }^{11}$. As a result, between August 2014 and June 2015, more than 2600 hectares, respectively 50 linear kilometres of the southern sector (between Danube and Arges rivers) of Limes Transalutanus were documented, in 67 aerial missions executed with UAVs, by recording, during 800 minutes of flight, more than 8300 vertical and oblique images from altitudes ranging between 100 and $300 \mathrm{~m}$, images further assembled into digital terrain models and orthophotos. Based on these images and their derivate products, a better spatial characterisation of the archaeological sites and structures belonging to the Roman limes known from previous researches was attained, while, in many cases, new features like watchtowers and roads were added to the general map of the ensemble ${ }^{12}$.

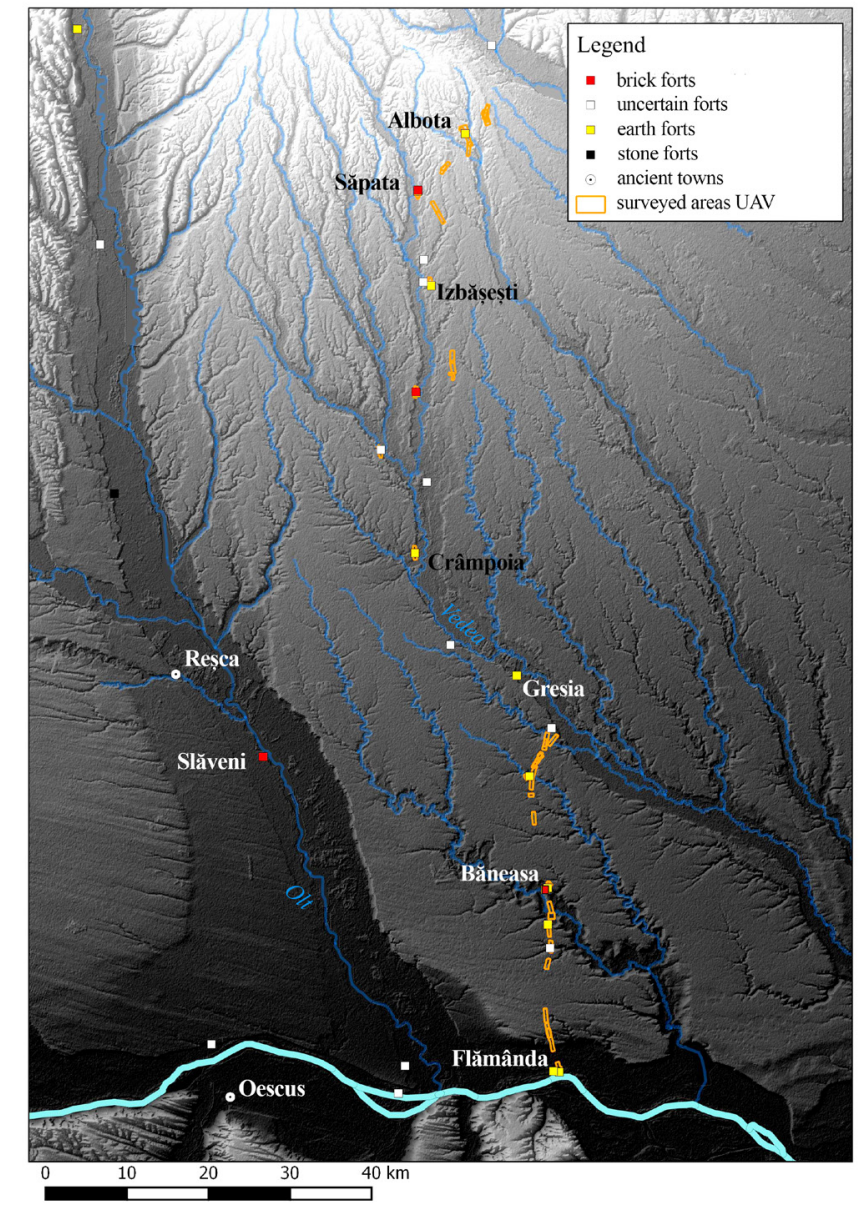

Fig. 1. Map of surfaces covered in UAV aerial mission (2014-2015) on the southern sector of Limes Transalutanus.

TEODOR/BEM/ȘTEFAN D. 2015

TEODOR 2016 
This makes the project the first large scale systematic use of UAV in Romanian archaeology, in tune with similar international endeavours, in particular focussed, not only on resolving archaeological issues, but on establishing efficient technical solutions: flying platforms, aerial navigation systems, data acquisition protocols, sequences of procedures for image photogrammetric processing, relevant in the field of medium and low altitude remote-sensing and aerial archaeology.

\section{DRONES AS PART OF A CHAÎNE OPÉRATOIRE} FOR ARCHAEOLOGICAL EXPLORATION

Prior to starting this project, along the southern sector of Limes Transalutanus only a single watchtower was known, a single civilian settlement was supposed at Movila Tătaru, while the layout of the Roman dyke was established only partially, based on older limited excavations and analysis of satellite and aerial imagery from the National Agency for Cadastre (ANCPI) ${ }^{13}$. None of its 13 known forts had been previously topographically surveyed. Under these conditions, the project investigation strategy was designed since the beginning as focused on solving the grave need to ensure the spatial documentation of sites, particularly dramatic if we take in mind the positioning of Limes Transalutanus inside the largest agricultural field of the country.

Efficiency, as in low-cost, rapidity and relevance, was a must, since the survey was intended to cover its entire southern extent (a corridor of $157 \mathrm{~km}$ in length, at least $400 \mathrm{~m}$ in width). The combination of selected investigations methods followed a progressive detailing of the spatial resolution in order to solve, not only the white spots (in which, for example, the layout of the Roman dyke was unclear), but also with the purpose of rapidly guiding the future, more laborious field research, towards the hot spots in need for detailing. The linear aspect of the site called for a particular augmentation of the investigation practices. These methodological adaptations should be relevant for the future research and heritage valorisation of large territories organized in linear layouts, along corridors, like those associated with the rescue archaeology of highways, road networks or utilities (gas pipes, various subterranean networks).

In short, in the Limes Transalutanus project, the technological purposes derived from the quest for providing spatial documentation in good resolution, but in efficient conditions, of archaeological sites and their surroundings, in order to make the transition from loose-resolution spatial data, openly available for large areas, like satellite imagery, historical cartography, ANCPI aerial imagery $(50 \mathrm{~cm} /$ pixel), to detailed site-level data, like distribution maps of artefacts found in systematic surface surveys, excavations plans and geological drillings. The extent of the archaeological area of interest in need for spatial documentation and the limited resources of the research project excluded from the start the possibility to use LiDAR or any other type of technology based on laser telemetry in order to obtain digital terrain models. Obviously, detailed topographic measurements with geodetic GPS and total station were taken in consideration only for very limited areas.

\footnotetext{
13 TEODOR 2015.
}

Instead, in order to cover this lack of intermediary resolution data and integrate site-level research in larger landscapes, the following triad of super-efficient investigation methods was tested:

- low and medium altitude ${ }^{14}$ aerial survey with remote controlled flying platforms/UAVs/drones used in efficient manner

- the complex processing of aerial images using Structure from Motion ${ }^{15}$ (SfM) algorithms with the purpose of obtaining ortophotographs and digital terrain models on region-sized sites at high resolution

- the extensive use of efficient geophysical prospection (mainly the method of measuring the magnetic soil susceptibility with the purpose of detecting and delineating archaeological sites or to establish its occupational patterns).

The combination between the portability and flexibility of multirotor flying platforms ${ }^{16}$, paired with the recent developments in the photogrammetric field which ensure the automatized production of digital terrain models without initial camera calibration, fulfilled our requirements for efficiency, low costs and relevance, delivering in the end orthophotos and terrain model with good resolutions $(5-25 \mathrm{~cm} /$ pixel). This aspect is significant not only because the resolution is higher than any other currently available data (fig. 2, 3); but, first of all, because it can be delivered for surfaces regardless of their extent and in any moment along the project timetable. In fact, this temporal availability proved to be, by far, the most significant advantage of the technology, especially when we have to deal with unpredictable funding, various institutional contexts or agricultural and meteorological factors. By opening the possibility to plan aerial missions, theoretically anytime, from any station point, with little regard to weather conditions (except for rain), to repeat the missions, adjust the route, flying altitude, photographing angle, all in real time, UAVs have become the main instrument to approach the archaeological landscape in the Limes Transalutanus project. If the advantages of using UAVs for archaeological sites' documentation are already recognized at international

\footnotetext{
14 The classification of flight altitudes' ranges may differ from one project to another. In this case, altitudes over $600 \mathrm{~m}$ were considered high altitudes, usually in the domain of regular aircraft survey, even if technically they can be reached by drones, too, but flight regulations forbid it; oblique images recorded from UAVs flying at medium altitudes $(200-600 \mathrm{~m})$ are best for site detection, while lower altitudes, under $200 \mathrm{~m}$, are good for vertical photographs and, thus, for site level aerial survey. In the case of smaller, more spatially concentrated archaeological sites, the range for UAV flight altitudes, considered low or medium, can be even lowered.

15 A procedure developed in the field of computer vision of extracting 3D data from sets of overlapping 2D images of the same scene acquired from multiple stations (camera in motion). Even if it is still a photogrammetric approach, in contrast with the traditional methodology, in the case of SfM, the positions of the cameras and their internal geometries are calculated by automatized algorithms, without any need for pre-requisites like camera calibration or use of in situ targets. Several software applications are already implementing these algorithms: AgiSoft Photoscan, PhotoModeller, SFMToolkit, Photosynth, Bundler, PMVS2, PIX4D, VisualSfM, 123DCatch, Drone2Map for ArcGIS. 16 After the general constructive type, drones can be divided into: fixed winged, mobile winged, with rotary motors, mixt or aircraft-like. Drones with rotary engines and propellers $(4,6,8$ or 12) have become favourites in the commercial sector due to their low production costs and good reliability. Such platforms, generally called multicopters, are those used in archaeology, also.
} 
Studies

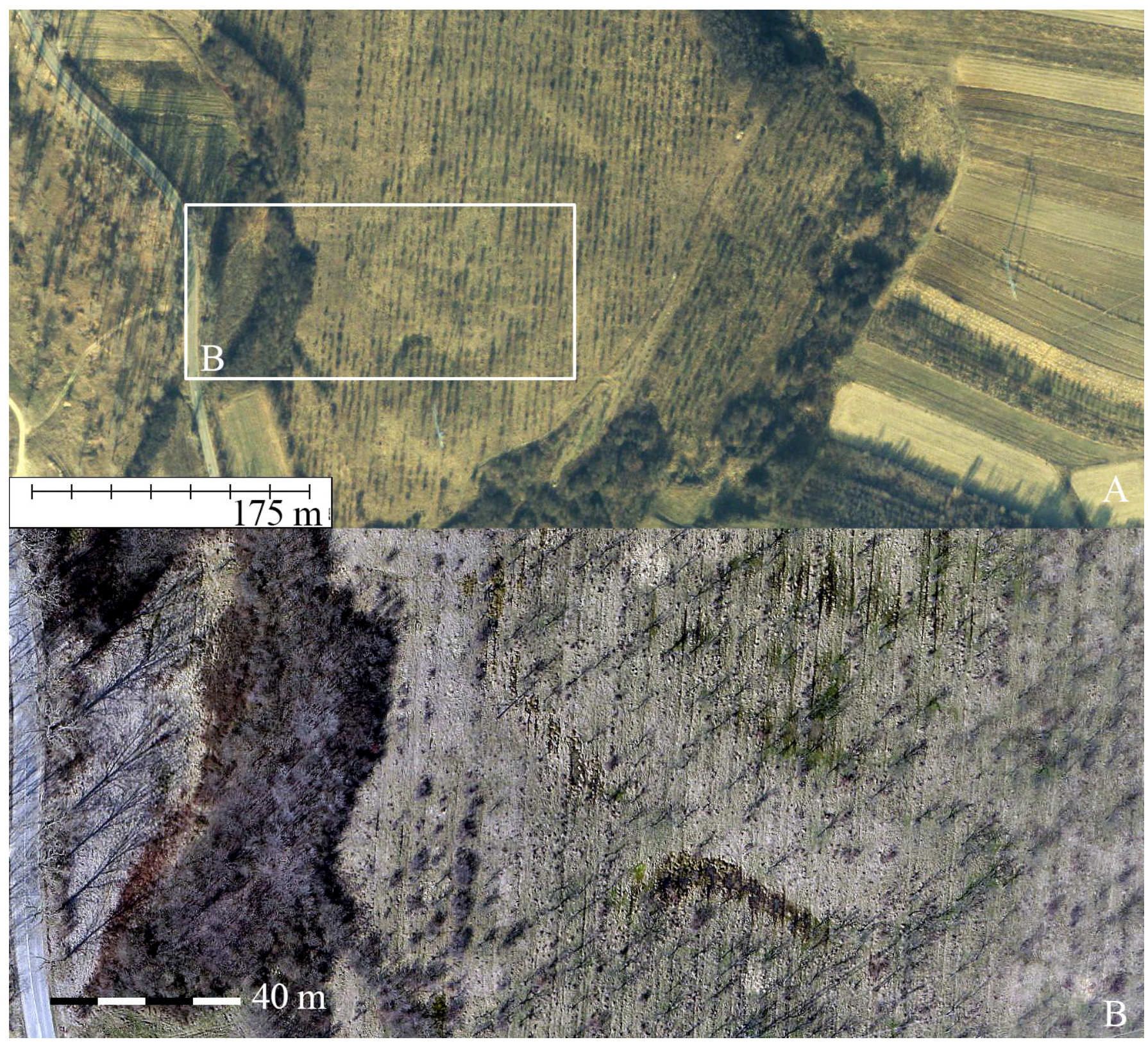

Fig. 2. Comparison between orthophotographs at maximum resolution: A (up) - image from ANCPI $50 \mathrm{~cm} /$ pixel; B - photo mosaic $17 \mathrm{~cm} /$ pixel based on images acquired with Canon PowerShot SX from 200 m altitude with Q1D2. Case study: Săpata forts.
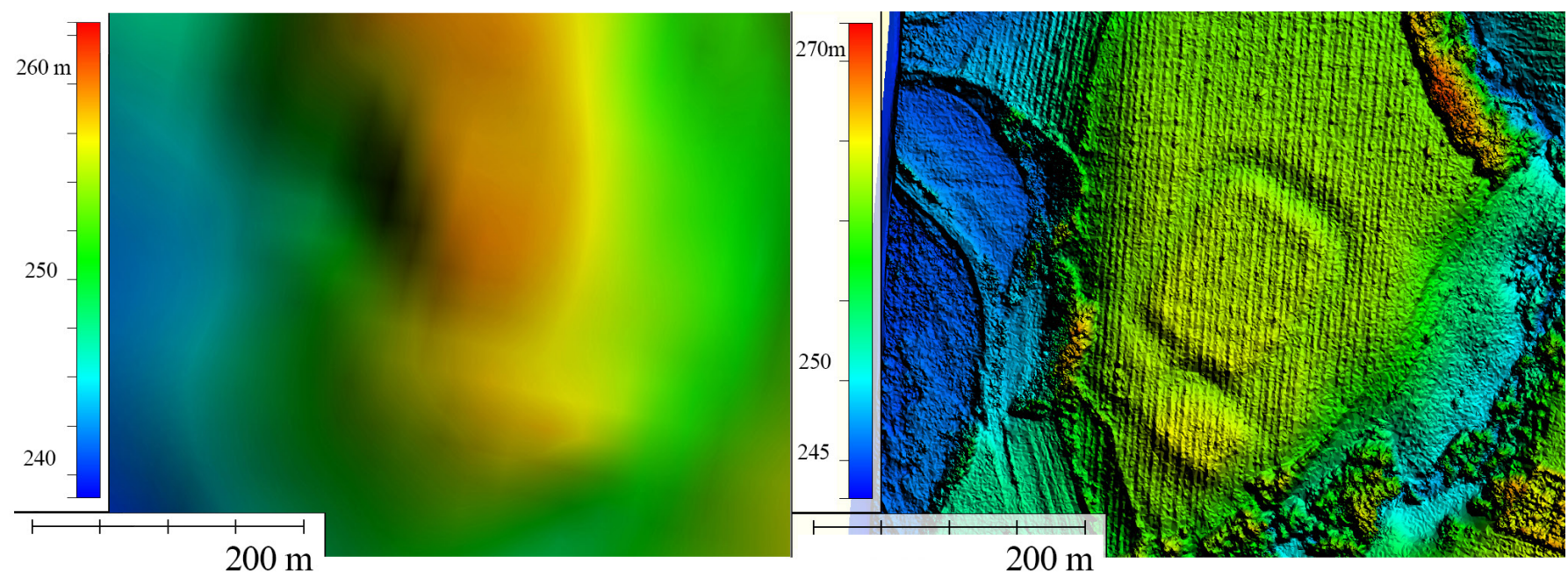

Fig. 3. Comparison between digital terrain models for Săpata forts area: left (DEM Europea/30 m resolution); right - 25cm/pixel resolution DSM obtained with SFM and Canon PowerShot SX camera carried by Q1D2 UAV. 
level, our experiment, run at the scale of an extended corridor site, more suitable for a regular aircraft survey, proved that the technology may be considered enough solid and trustful even in large scale aerial investigations.

\section{TESTED FLYING PLATFORMS}

The initial tests on Limes Transalutanus ${ }^{17}$ were carried on with a hexacopter designed and built by the authors in a previous research project ${ }^{18}$ with the purpose of surveying individual/delimited archaeological sites. The H1D1 prototype has a $1.2 \mathrm{~kg}$ payload (600 $\mathrm{g}$ for the 2-axis brushless camera stabilisation system, $200 \mathrm{~g}$ for the damping vibration component and $400 \mathrm{~g}$ for the photo camera and lenses). Its total take-off weight is $4.2 \mathrm{~kg}$, and the total flight time in real atmospheric conditions, with $10.000 \mathrm{mAh}$ LiPo batteries, is 11 minutes. A special feature of its navi-controller is the ability to program the aerial missions, regardless of take-off direction or base station. Following this strategy, a regular single-site mission progresses around the site's centre (used as take-off station), ensuring terrain coverage with a mosaic of vertical images on a circular layout with an approximatively $400 \mathrm{~m}$ radius. The resulting orthophotography at $5 \mathrm{~cm} /$ pixel resolution and digital terrain model $(25 \mathrm{~cm} /$ pixel) describe a 40 ha surface. In addition, a set of oblique images recorded on the same occasion ensure visibility coverage on a $1000 \mathrm{~m}$ radius starting from the take-off position.
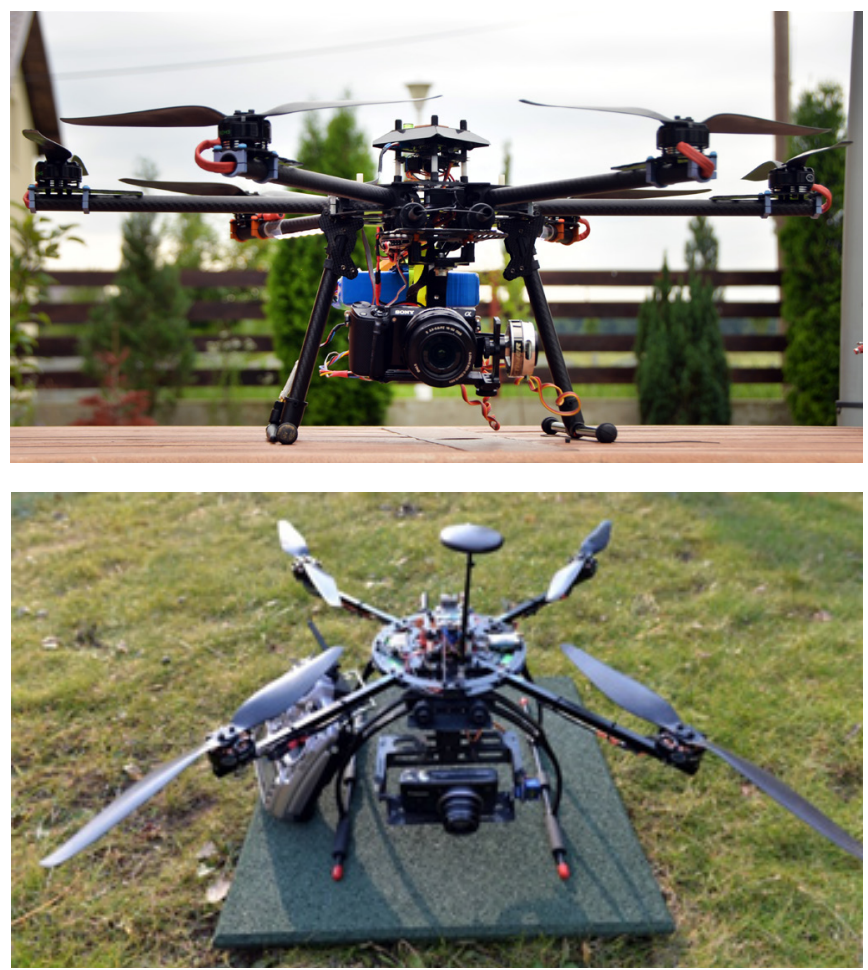

Fig. 4. UAV platforms developed in the project: up - H1D1; down Q1D2

Soon enough, aerial tests had revealed that the H1D1 hexacopter ${ }^{19}$ did not fulfil the efficiency requirements raised

\section{TEODOR/STEFAN M. 2014, p. 36. Fig. 2 \\ PNCDI PN II - 69/2011.}

19 The number of rotary engines in a drone is implemented after taking in consideration several factors. If it uses more engines, a platform will be able to lift higher payloads, but the complexity and costs of the model will increase. Another significant aspect is redundancy. A quadcopter (4 rotary engines and 4 propellers) cannot fly in case one engines stops or one propeller brakes. A by the site's linear layout. Accordingly, a new platform was developed following the optimisation of several parameters. Among these, reducing significantly the take-off weight was expected to ensure a better portability, mandatory in order to increase the transport capacity in heavy terrain conditions (lack of access roads, extended wet lands) diminishing the limitation of not being able to easily reach the site centre for take-off. In addition, reducing weight contributes to the minimization of either direct or collateral damages in case of crash. Lengthening the flight-time, but with LiPo batteries with smaller capacities for lesser $\operatorname{costs}^{20}$ and reduced weight, was also an objective. Not least, ensuring remote-control on large distances (up to $1.5 \mathrm{~km}$ ) and the redundancy of this control were considered of critical importance. The resulting prototype (Q1D2) was developed on a quadcopter platform, lighter and more efficient than copters using more engines. In order to select the most suited thrusting solution there had been tested over 300 combinations of engines, propellers and electronic speed controllers (ESC). The final solution consisted of brushless engines with a small height/ diameter ratio (pancake type), propeller made of carbon fibre (diameter: 13 inch, pitch 4.5 inch, custom profile) and ESC modules with an ultra-rapid response capacity. The total payload of this platform is $600 \mathrm{~g}$ (140 $\mathrm{g}$ for for the 2-axis servo camera stabilisation system, $60 \mathrm{~g}$ the damping vibration component and $400 \mathrm{~g}$ camera and lenses). The total weight at take-off is of only $2.1 \mathrm{~kg}$ and maximum safe flighttime in real atmospheric conditions with LiPo $5300 \mathrm{mAh}$ is 15 minutes. The Q1D2 is equipped with complex control and navigation modules, being able to complete aerial missions in one of the following ways (even combine flight types): manual flight control and manual navigation, manual flight control and instrumental navigation (real-time tracking of a flight route on a map and real time visualisation of the terrain through the on-board camera video downlink), automatic flight control and autonomous navigation. The multilevel control system consisting of: 2-way remote control radio with frequency hopping spread spectrum technology (FHSS), instrumental telemetry and navigation (also based on FHSS) and video downlink radio (FPV - first person view) guarantee a high level of redundancy. Essentially, in the event any of the control and navigation subsystems malfunctions, the capacity for mission flight and returning to home remains intact, based on the overall system redundancy. Another vital component (the fail-safe system) manages more complex malfunction scenarios, like the exhausting of the energy supply or the operator losing its orientation. After hexacopter system is still able to hover if such accidents should occur, with the condition that its total weight does not exceed the maximum payload for the number of remaining functioning propelling elements. Nevertheless, controlling a flawed hexacopter remains difficult as it will tend to rapidly rotate around its vertical axis. Octocopter systems have a better redundancy, allowing the continuity in mission in case one or even two of its propulsion elements brake. However, despite better redundancy, hexacopters and octocopters have a smaller energetic efficiency, which is a critical requirement in case of large-scaled surveys.

20 The LiPo batteries used for the Unamend Aerial Vehicles propulsion represent a significant component of the platform initial costs, but also of its exploitation period expenses. Their rather small life expectancy (optimum 12 months since production) is often a neglected detail. After 1 year they should be used only for at most another year as back-up. If we add to this the need for many batteries in the field (minimum 4, optimum 6), the high exploitation costs became more understandable. 
Table 1

\begin{tabular}{|llll|}
\hline UAV & H1D1 & Q1D2 & DJI Phantom 3 \\
Propulsion system & Hexacopter & Quadcopter & Quadcopter \\
Maximum flight speed & $18 \mathrm{~m} / \mathrm{s}$ & $20 \mathrm{~m} / \mathrm{s}$ & $16 \mathrm{~m} / \mathrm{s}$ \\
Maximum lifting speed & $5 \mathrm{~m} / \mathrm{s}$ & $5 \mathrm{~m} / \mathrm{s}$ & $5 \mathrm{~m} / \mathrm{s}$ \\
$\begin{array}{l}\text { Damping camera vibration system } \\
\text { (gimbal) }\end{array}$ & 2 axes, brushless & 2 axes, servo & 2 axes, servo \\
Payload & $1.2 \mathrm{~kg}$ & $0.6 \mathrm{~kg}$ & $0.3 \mathrm{~kg}$ \\
of which: gimbal weight & $800 \mathrm{~g}$ & $200 \mathrm{~g}$ & $200 \mathrm{~g}$ \\
camera weight & $400 \mathrm{~g}$ & $400 \mathrm{~g}$ & $100 \mathrm{~g}$ \\
Total take-off weight & $4.2 \mathrm{~kg}$ & $2.1 \mathrm{~kg}$ & $1.3 \mathrm{~kg}$ \\
Batteries capacity & $10600 \mathrm{mAh}$ & $5300 \mathrm{mAh}$ & $4480 \mathrm{mAh}$ \\
Flight time in real conditions & $11 \mathrm{~min}$ & $15 \mathrm{~min}$ & $18 \mathrm{~min}$ \\
Camera & SonyNex5T & Sony & FC300S $5 \mathrm{~T}$ \\
Resolution & $16 \mathrm{Mpx}$ & $16 \mathrm{Mpx}$ & $12 \mathrm{Mpx}$ \\
Sensor size & $23.4 \times 15.6 \mathrm{~mm}$ & $23.4 \times 15.6 \mathrm{~mm}$ & $6.17 \times 4.55 \mathrm{~mm}$ \\
Lenses & interchangeable & interchangeable & fixed \\
\hline
\end{tabular}

the execution of numerous flights in different atmospheric conditions (from calm to strong winds) during the second semester of 2014, the model was ready to be used in the spring of 2015.

If during the initial test-flights, completed with a multicopter platform configured as a hexacopter (H1D1 prototype) with a maximum payload of $1.2 \mathrm{~kg}$, the flight time ranged between 7 and 12 minutes (depending on the used battery capacity), once the flight platform was updated, the executed missions got longer, up to 15 minutes. The use of a lighter prototype (Q1D2) and the optimisation of the land covering strategy offered the possibility to document through orthophotography and digital terrain model, as a result of a single flight mission, a surface ranging from 50 to 90 hectares, which, if related to the linear character of the archaeological target, may be translated into a corridor of $450 \mathrm{~m}$ in width and $1.5 \mathrm{~km}$ in length.

In the autumn of 2015, when the main stage of aerial survey by UAV had been already completed, we tested, in the commercial category, one of the most popular platforms amongst video enthusiasts, the DJI Phantom series-in its $3^{\text {rd }}$ release. The model has a small weight (less than $1.3 \mathrm{~kg}$ ), a rather extended flight time capacity (up to 18 minutes in real atmospheric conditions), integrated photo-video camera, damping vibration and stabilisation system on 3 axes with excellent performances, digital video downlink allowing FPV navigation (with some latency though). The tests prove that the model can be used with reasonable results for archaeological purposes, either delimited sites or corridor applications, especially in those cases where portability is the first valued requirement. Resulted images have a medium to low quality, but can be processed in SfM procedures. An advantage is the good quality of videos. However, currently DJI Phantom series is not suitable for complete autonomous aerial missions. Even more, the instrumental navigation based on telemetry is not enough accurate, the system being prone to slight but noticeable heading deviations.

\section{TESTED OPTICAL SENSORS}

The main component of the aerial exploration of archaeological sites is their image and/or video recording from above with the purpose of detecting new structures, documenting, spatially describing or just illustrating the heritage. This is why the selection of photo equipment, especially of the camera's sensor and optical system is of key importance, but in the case of air-carried equipment, this choice will be always done depending, not just on their quality ,but also on their weight. The choice of the photo solution is actually a compromise taking in consideration: the sensor's size, the resolution, the lenses' quality (resolving power, distortions, maximum aperture) and the weight of the entire ensemble, calculated in relation with the flying capacity of the UAV and its maximum payload. Other ingredients of this formula are the flight altitude, the size and shape of the targeted site.

The main products of aerial archaeology carried on with UAVs at low and medium altitudes can be categorized as in Table 2.

Ideally, the best lenses are the fixed ones, not electronically corrected, normal or wide (in the range 35 $50 \mathrm{~mm}$, full frame format equivalent), with well controlled linear and, especially, nonlinear distortions. In real practice, the UAVs' requirements call for a different optical solution. First of all, the flying altitude should be lowered, because high altitude surveys are becoming more and more legally restricted, while they oblige to a considerable energy expense. A lower flight altitude leads, however, to smaller area coverage for each photo. This, in turn, forces to using 


\section{Primary Data Sets}

Vertical aerial images (nadir - the optical axis is perpendicular on the terrain surface); advantages: constant scale, maximum details, minimum distortions

Oblique aerial images with low angle (smaller than 45 degrees, in which the horizon is not visible), known also as low-oblique images; they are taken in consideration together with vertical data sets, during the Structure from Motion workflow, in order to determine the internal camera geometry

Oblique images with high angle (in which the horizon is visible), known as high-oblique images; they are useful for the detection of anomalies in soil and vegetation; offer an integrating perspective over the landscape and features

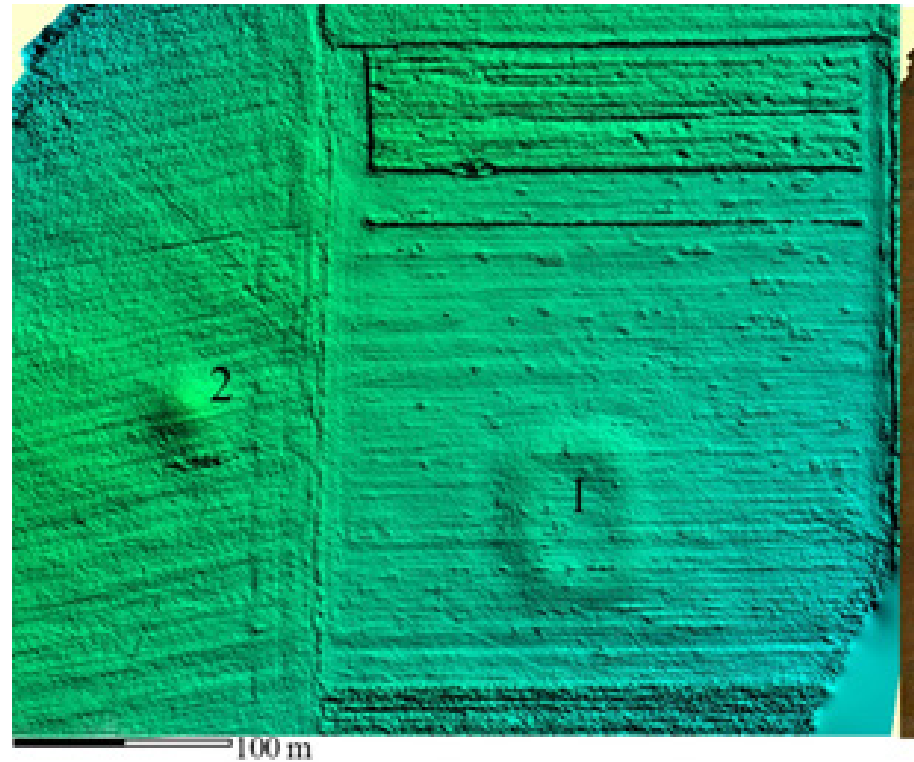

Secondary Data sets (derivate)

Orthorectified mosaic of vertical images (resolution $5 \mathrm{~cm} /$ pixel), obtained by implementing photogrammetric Structure from Motion algorithms; for acceptable results it is necessary to document the same scene in at least 3 images, and for excellent results in 5-9 images

The digital model of the terrain (digital surface model - contains also the elevation level of the vegetation and anthropic structures); it is calculated based on a mix of vertical and low-oblique images using SfM algorithms

\section{The digital elevation model of the terrain, a bare-earth type of} DSM, obtained by using automatized or semi-automatized classification procedures to the dense point clouds; with resolutions higher than $25 \mathrm{~cm} /$ pixel; the practice has shown that the observation of digital terrain models illuminated under various angles represents the most successful method for the detection of archaeological features; the method is often more relevant than the analysis of aerial imagery (fig. 5)

- topographic plans at various scales, with or without contour levels - elevation profiles

- visibility analyses and other spatial analyses that use the high resolution digital terrain models

Fig. 5. Sometimes digital terrain models have a better revealing power, as for example here, for the case of the small fort from Băneasa (1) represented in DSM (left) and orthophoto (right); 2 - tumulus.

wider lenses (ultra-wide, 20-28 mm) known for their greater perspective distortions and heavier weight (for example, the Zeiss Milvus $21 \mathrm{~mm} \mathrm{f/2.8} \mathrm{ZF} 2$ in Nikon F-mount or Canon EF-mount, weights 755 grams). Perspective errors cannot be compensated in the post-processing phase, the only solution to their minimization being to fly at higher altitudes above forested areas or tall buildings. Weights can be reduced by diminishing the optical quality, either by selecting smaller sensors (in this way, both the image circle and total weight become smaller $)^{21}$ or by selecting lenses with a more closed maximum aperture (this makes the lenses less heavier, which is good, the depth of field to increase, again a good thing,

\footnotetext{
${ }_{21}$ In this case one should take in consideration the crop factor for the used
} sensor in relation with the full frame format of $36 \times 24 \mathrm{~mm}$. For example, an APSC sensor $(24 \times 18 \mathrm{~mm})$ has a surface by $1.5 \mathrm{x}$ smaller than a full frame (crop factor $=1.5$ ). Therefore, using a $16 \mathrm{~mm}$ lenses on APSC will have the same effect as a $24 \mathrm{~mm}$ lenses on a full frame sensor $(16 \mathrm{~mm}$ x $1.5=24 \mathrm{~mm})$. but also this will lead to a smaller quantity of light to reach the sensor and that is not so good). In the end, the optical qualities of the lenses will suffer: the quantity of light is reduced on the image's periphery (vignette), resolution and resolving power are kept only in the image's centre and the geometrical distortions are considerable. With the condition of acknowledging these shortcomings, the final obtained weight can be thus, significantly diminished. For example, the Sony $16 \mathrm{~mm} \mathrm{f} / 2.8$ pancake lenses for APSC sensor camera weighs only 70 grams, while lenses for cameras with smaller sensors (for example 1/ 2.3 inch) weigh even less, few grams. Obviously, reducing size results in loosing quality: lower sensibility (limits their use only in very good light conditions, not at dusk or in early morning or thick clouds), low color depth (altered chromatic accuracy) and reduced dynamic range (poor results in high light or strong 


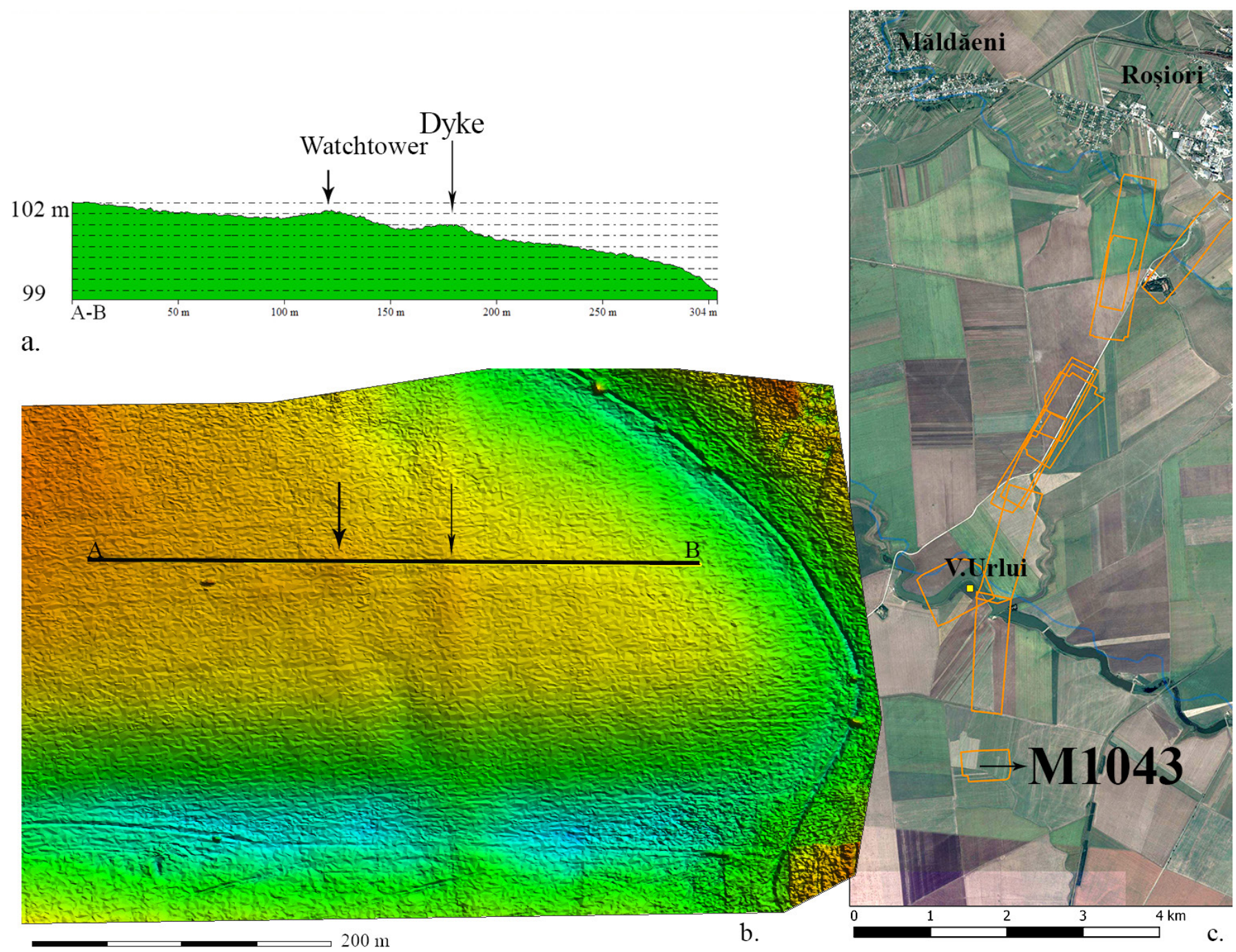

Fig. 6. DSM (b) and elevation profile (a) for the dyke and the new watchtower identified south from Valea Urlui at Epureasca in aerial mission M1043.

contrast situations). In the last generation commercial cameras, the linear and nonlinear distortions are strongly electronically corrected (in fact, masked). This adds an extra level of geometrical distortion which has to be compensated during the photogrammetric processing and which can cause erroneous results in the automated deduction of the internal camera geometry ${ }^{22}$.

For the Limes Transalutanus project we tested several cameras, with either fixed or interchangeable lenses. Finally, for the actual surveys we selected three: two compacts with small sensor (1/ 2.3 inch diagonal, $6.17 \times 4.55 \mathrm{~mm}$ ) - Canon PowerShot SX260 and FC300S from DJI Phantom series 3 and a third one - mirrorless, Sony NEX-5T with a 23.4 x $15.6 \mathrm{~mm}$ sensor. The Sony was paired with two lenses: a Sony zoom ( $16 \div 50 \mathrm{~mm}, \mathrm{f} / 3.5-5.6)$ blocked in $\mathrm{f}=16 \mathrm{~mm}$ (equivalent with full frame $\mathrm{f}=24 \mathrm{~mm}$ ) and a fixed $19 \mathrm{~mm}$ Sigma, $\mathrm{f} / 2.8$ ( $\mathrm{f}=28.5 \mathrm{~mm}$ on full frame). We did not used DSLR cameras in order to avoid the supplementary weight of the optical viewfinder with penta-prism or mirror - a facility

22 Despite the recommendations stated in all the integrated applications' user guides, the Structure from Motion and Multi View Stereo techniques are quite robust, accepting images characterized by a high degree of optical imprecision. Nevertheless, there is a certain critical amount of compromises manageable by the systems. After a certain point, the models cannot be computed or the errors are too great. with no application in UAV based aerial archaeology. The Sony NEX-5T has proved to be a very good solution, with its relatively large sensor (APSC, crop factor 1.5) and large dynamic range, which at the weight of only 276 grams for the body is one of the lightest mirrorless, and considerably less heavy than a DSLR with a similar type of sensor.

\section{PLANNING AERIAL MISSIONS}

The linear layout and considerable size of our targeted site - Limes Transalutanus imposed a specific approach to establishing aerial survey strategies. The first stage should be always about deciding on take-off stations by considering site accessibility. We looked for those locations reachable by off-road vehicles, positioned either in the center of the area to survey or in an extremity of a linear sector. However, bearing in mind the $1.5 \mathrm{~km}$ distance as the maximum to be efficiently covered in a single mission, take-offs, if not possible otherwise ${ }^{23}$, were sometimes located at quite some distance from the target. The best strategy was to find a take-off station that allowed a double survey, meaning the execution of two missions in opposite directions, thus,

\footnotetext{
23 The direct access in the field was quite often problematic, as during spring - the best season for aerial survey, the agricultural lands are very humid and muddy.
} 
completing sectors as long as 3 kilometers.

Because flights were usually made at distances over $1 \mathrm{~km}$, direct observation of UAV for flight operator's orientation is not possible, nor the UAVs navigation based on video downstream link is relevant, due to uniform, confusing, textures of agricultural fields or forest, and lack of clear visibility of archaeological targets. Instead, targets are approached by using a combination of free flight based on reading and instant interpreting of telemetry data (this is quite efficient for linear missions, using only data regarding heading, altitude, distance and speed; of course, some flight experience is needed for this) and orientation on an electronic map showing in real time the current position of the UAV (this can be also done with a second operator giving instructions); still, LCDs in strong outdoor light remain difficult to observe. Else, aerial surveys ca be totally preprogrammed. This is a good option if the take-off station is well known and accessible.

The maximum efficiency in flight is attained, for linear missions, by using a back and forth pair of tracks ${ }^{24}(1.5 \mathrm{~km}$ in length) distanced at $200 \mathrm{~m}$, parallel with the main path of the dyke. Even if for best optical quality (smallest distortions, best resolution) of derivate products (orthophotos, digital terrain model) the target should be recorded in the central part of the image, for the most efficient solution in cases of corridor layouts, it can be acceptable to fly on tracks positioned above the sides of the site, in order to cover a larger area, up to $450 \mathrm{~m}$ wide. Our regular flight altitude was around $200 \mathrm{~m}$. For good results in the post-processing phase the images (vertical) have to overlap to a certain degree. This is of $80 \%$ along the flight track and 60\% between tracks (lateral). Also, in order to improve the quality of the automatic calculation of the internal camera geometry (in the bundle adjustments procedure) low-oblique photos can be added to the set of vertical images regularly used for the mosaic. Oblique images can be recorded during the same mission organized for vertical coverage (one or two 360 degree

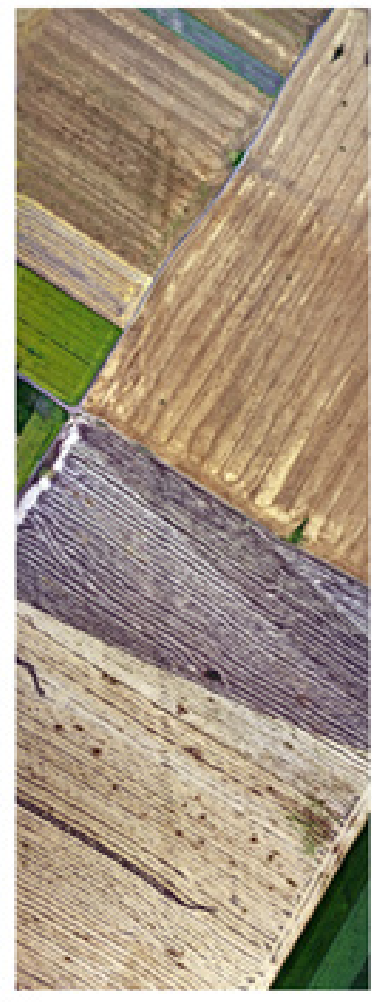

a. rotations, and/or oblique images recorded during the takeon and take-off sequences) or in a dedicated one.

Even if in the beginning of the survey we used targets and accurate measured them by total station or geodetic GPS, it soon become obvious that this common georeferencing procedure ${ }^{25}$ was too time consuming (placing targets on the entire track $1.5 \mathrm{~km}$ in length, $400 \mathrm{~m}$ width, in the middle of extended fields or forests) in relation with the efficiency of aerial survey, especially considering that sites were not easily accessible, either by car or by foot. Therefore, we opted for lesser accurate georeferenced products (1-5 m on XY $)^{26}$ by using GPS data supplied by the UAV's sensorial system, inserted into the images EXIF files. This additional data helped also to speeding up of the identification of tie points in image sets, by diminishing the number of iterative image comparisons. This procedure, however, does not allow a consistent integration of elevations acquired in different missions on adjacent areas.

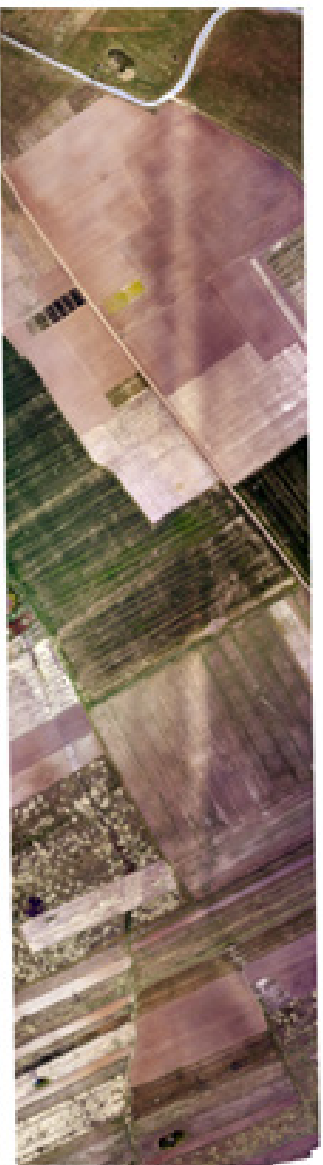

b.

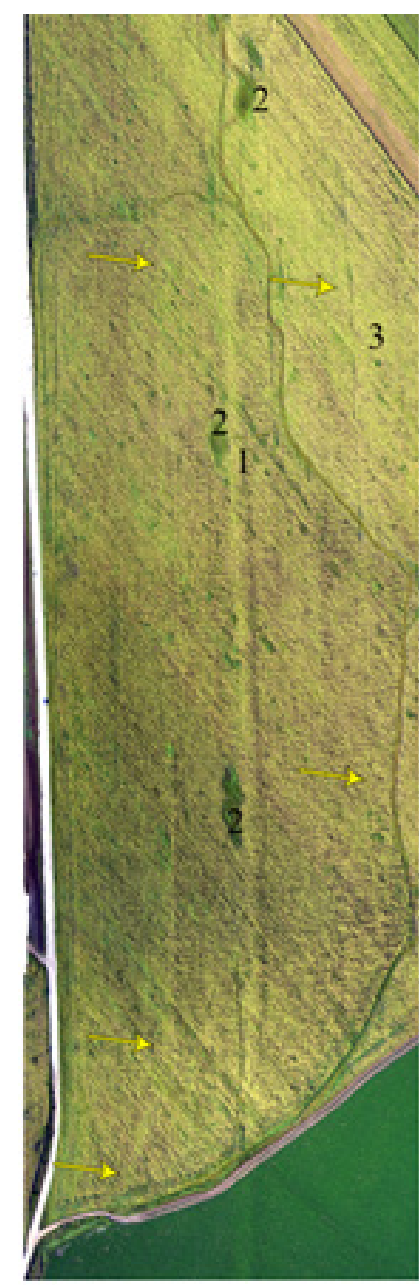

c.
Fig. 7 Examples of aerial field marks for the Limes Transalutanus dyke in mosaics of vertical images: b. Poiana Roșie, c - north of Valea Mocanului: 1 - the dyke, 2 - vegetation marking lowered terrain, 3 - (between yellow arrows) modern underground utilities.

\begin{abstract}
5 Nevertheless, it still remains the recommended procedure for nucleus/ individual sites, like forts for example. Orthophotos and digital terrain models are initially created in SfM workflows in an arbitrary reference system; they are not scaled and have no orientation. These can be added later to the final 3D block, using reference points, minimum of 3 best in the range of $9-12$ or by initial uses of GPS data added to the images' EXIF files.

${ }_{26}$ This could get improved very quickly by the future integration of RTK GPS on UAVs
\end{abstract}




\section{SfM WORKFLOWS}

Two approaches were tested: an integrated commercial solution (Agisoft Photoscan) with good results, but with limitations in controlling parameters' definition especially in what concerns the texturing and georeferencing and an open source modular sequence starting from VisualSfM ${ }^{27}$ (for detecting invariance points, creation of sparse point cloud), followed by employment of CMVS/PMVS2 ${ }^{28}$ for generating dense clouds, and then by different software for mesh generation and texturing $\left(\mathrm{MeshLab}{ }^{29}, \mathrm{SURE}^{30}\right.$ or even Global Mapper, which is commercial software).

The integrated solution is easy to use, however managing large data sets is conditioned by the quality of the used hardware. The application uses intensely not only the PC's processor and memory, but also the graphic component. Powerful graphic stations are needed. For lower costs, gaming platforms can be taken as an alternative. In order to be as efficient as possible, the quest for a minimal solution with acceptable results was done in the processing part, as well. For example, after some comparative tests, we decided that the quality differences between medium and high quality dense clouds are insignificant at the scale of a landscape analysis, almost in all cases covered by some sort of vegetation, not enough to justify the considerable longer processing times, even if high equals 4 times denser clouds than medium (in Photoscan). Similarly, in order to shorten working times and avoid computer blockage, we searched for a minimal optimal number of photos to be aligned in a single event. Even if in theory more photos equals better camera geometry and $3 \mathrm{D}$ terrain model, in practice, the quality of used images is more important; also, good results can be reached with a critical amount of images, after which any addition does not significantly improves the solution. In our case, we estimated that a batch ranging between 90 to 140 images may give relevant results for a 40 to 70 hectares area covered in one mission. As main problems we noticed, first of all, the impossibility to decide on what image and what part of it is selected for the mosaic and, secondly, the tendency of terrain models calculated for areas with small elevation differences to suffer of the dome effect.

The use of a modular solution, like the pair of VisualSfM and PMVS2 has the advantage of being free software; however, the level of required specialization is higher and could result in costs for professionals to get involved or trained. Nevertheless, for ultra-specialized applications or for solving difficult problems it may be good choice as it allows the integration of additional modules developed by independent researchers. For example, uniform textures like forests and agricultural fields do not provide enough chromatic details to help the successful identification of tie points. This could be corrected by employing a specific

\footnotetext{
27 Developed by Changchang Wu; free for personal, academic use (http:// ccwu.me/vsfm/)

28 CMVS - Cluster Multi View Stereo; PMVS - Patch-based Multi-View Stereo ( http://www.di.ens.fr/pmvs/)

29 A very popular open source application used for generation and editing of 3D surfaces (http://meshlab.sourceforge.net/)

30 SURE - Photogrammetric Surface Reconstruction from Imagery, ROTHERMEL et al. 2012.
}

disambiguation algorithm ${ }^{31}$

An important aspect for the archaeological aerial remote-sensing is generally linked with vegetation, especially with its filtering in order to uncover hidden archaeological structures, or just the bare-earth for high-resolution elevation models. While LiDAR is the established solution, SfM applied to images acquired from drones can offer a kind of alternative. For example, dense cloud points can be codified according to the variation of degree of inclination on a certain distance. Photogrammetry is obviously not an active high-density measurement method like LiDAR, so it cannot reveal things under a canopy, but by employing certain procedures, areas with high vegetation or buildings can be ruled out from the mesh model calculation, which in cases is enough useful. Further developments in the field of dense point clouds editing and codification could boost the usefulness of this low-cost technology

\section{FINAL THOUGHTS}

Presently, there isn't available an optimized and completely integrated workflow for low and medium aerial archaeology. This is why workflow stages should be evaluated and customized on individual basis. Professional UAVs are generally tailored for video recording and that is why they are heavy, inefficient for larges surveys and dangerous in case of accident. Commercial platforms developed for mass sale are portable and easy to use, but they still do not have advanced navigation capabilities to execute autonomous missions on enough large distances, while their cameras are characterized by a considerable amount of optical compromises. The more suitable available commercial equipment, rather small in number, are those designed for cadastral and geodetic activities. Many of these are heavy platforms and quite expensive (up to 10.000 dollars) and their cost may not be justified, as there is no need for such high resolution in case of large-scale archaeological aerial survey. We consider that the development of custom-made flying platforms remains, for the moment, the most efficient and the lowest cost solution. The use of cameras and specially designed photogrammetric optics (distortion free) is not possible on multicopters due to considerable weights ${ }^{32}$. That is why commercial cameras have to be used. Choosing a certain model is done as a compromise between quality and weight depending on targeted site type. Flight strategy has a significant impact on the final quality of orthophotos and terrain models. Deciding on the appropriate model of terrain coverage should be done according to site shape, size and required data resolution. Once recorded and available for individual analysis or as data sets (mosaics of images) aerial images, vertical and oblique, can be processed through SfM and MVS algorithms into orthophotographs and digital terrain models. In this part of the process, in case of large data sets with an iterative character, an integrated solution can be more appropriated, while for challenging situations, modular software could help to have more access to parameters and source code.

Having in mind the rapid evolution in this field, we

\footnotetext{
WILSON/SNAVELY 2013

32 For example, Leica RD 30 airborne camera, with Leica Nag-D f/50 mm lens and memory module, weights more than $4.4 \mathrm{Kg}$.
} 
expect that, in short time, many integrated solution to become available and at affordable costs. It remains to be seen how close they will get to meet the requirements of large scale systematic archaeological research.

\section{REFERENCES}

\section{BERECKI/CZAIJLIK 2012}

Berecki, S./Czaijlik, Z., Panorame istorice, Târgu Mureș: L'Harmattan/Mega, 2012.

BERGER et al. 2014

Berger, M./Tagliasacchi, A./Seversky, L./Alliez, P., Levine, J./Sharf, A./Silva, C., State of the Art in Surface Reconstruction from Point Clouds, Eurographics - State of the Art Reports, Strasbourg, France, 1 (1), 161-185.

CONCEPT FOR OPERATIONS OF DRONES 2015 https://www.easa.europa.eu/system/files/dfu/204696_ EASA_concept_drone_brochure_web.pdf

FERNÁNDEZ-LOZANO/GUTIÉRREZ-ALONSO 2016

Fernández-Lozano J./Gutiérrez-Alonso, G., Improving archaeological prospection using localized UAVs assisted photogrammetry: An example from the Roman Gold District of the Eria River Valley (NW Spain, Journal of Archaeological Science: Reports 5, 509-520.

FERNÁNDEZ-HERNANDEZ ET AL. 2015

Fernández-Hernandez, J./González-Aguilera, D./ Rodríguez-Gonzálvez, P./Mancera-Taboada, J., ImageBased Modelling from Unmanned Aerial Vehicle (UAV) Photogrammetry: An Effective, Low-Cost Tool for Archaeological Applications, Archaeometry 57, 128-145.

FURUKAWA/PONCE 2010

Furukawa, Y./Ponce, J., Accurate, dense, and robust multiview stereopsis, EEE transactions on pattern analysis and machine intelligence 32 (8), 1362-1376.

LOWE 2004

Lowe, D.G., Distinctive Image Features from Scale-Invariant Keypoints, International Journal of Computer Vision 60(2), 91-110

MOZAS-CALVACHE ET AL. 2012

Mozas-Calvache A.T.,/Pérez-García J./Cardenal-Escarcena F./Mata-Castro E./Delgado-García J., Method for photogrammetric surveying of archaeological sites with light aerial platforms. Journal of Archaeological Science 39 (2), 521-530.

OLTEAN 2007

Oltean, I., Dacia: Landscape, colonisation, romanisation, Routledge, 2007.

PALMER/TÂRNOVEANU/BEM 2009

Palmer, R./Oberländer-Târnoveanu, I./Bem, C., Arheologie aeriană în România și în Europa, București, CIMEC, 2009.

REMONDINO/FRASER 2006

Remondino, F./Fraser, C., Digital Camera Calibration Methods: Considerations and Comparisons, International Archives of Photogrammetry, Remote Sensing and Spatial Information Sciences 36(5), 266-272.

REMONDINO ET AL. 2014

Remondino Fabio, Spera Maria Grazia, Nocerino Erica, Fabio Menna, Francesco Nex 2014 - State of the art in high density image matching, The Photogrammetric Record, 2014, 29, 144-166.

REPORT CODE: SE 3099

UAV Drones Market by Type (Fixed Wing, Rotary Blade, Nano, Hybrid), Application (Law Enforcement, Precision Agriculture, Media and Entertainment, Retail), \& Geography (Americas, Europe, APAC, RoW) - Analysis \& Forecast to 2020 by: marketsandmarkets.com, Publishing
Date: October 2015, Report Code: SE 3099 (supplied at request by marketsandmarkets.com).

ROTHERMEL et al. 2012

Rothermel, M./Wenzel, K./Fritsch, D./ Haala, N., SURE: Photogrammetric surface reconstruction from imagery, Proceedings LC3D Workshop, Berlin 8.

RUS 2015

Rus, G.E., Aerial Archaeology in Romania. Sites from Roman Dacia examined using aerial photos, Journal of Ancient History and Archaeoloy 2 (4), 2015.

TEODOR 2015

Teodor, E.S., The Invisible Giant: Limes Transalutanus. An overview south of Argeș River, Târgovişte: Cetatea de Scaun, 2015.

TEODOR 2016

Teodor, E.S. (ed.), Arheologia peisajului și frontierele romane, Târgoviște: Cetatea de Scaun, 2016, forthcoming.

TEODOR/STEFAN D. 2014

Teodor, E.S./Ștefan, D., Technological Challenges on Limes Transalutanus, Antiquity Projects Gallery, December 2014.

TEODOR/ȘTEFAN M. 2014

Teodor, E.S./Ștefan, M., Landscape Archaeology along Limes Transalutanus, Journal of Ancient History and Archaeoloy 1(3), 2014

TEODOR/BEM/ȘTEFAN D. 2015

Teodor, E.S./Bem, C./Ștefan, D., A story about one shot from three thousand five hundred, AARgNews 51, September 2015, 25-31.

TRIGGS et al. 2000

Triggs, B./McLauchlan, Ph./Hartley R.I./ Fitzgibbon, A.W., Bundle Adjustment - A Modern Synthesis, Vision algorithms: theory and practice. Springer Berlin Heidelberg, 298-372.

WILSON/SNAVELY 2013

Wilson, K./Snavely, N., Network Principles for SfM: Disambiguating Repeated Structures with Local Context, The IEEE International Conference on Computer Vision (ICCV), 2013, pp. 513-520.

ZHANG et al. 2012

Zhang, Sh./Qu, X./, Ma, Sh./Yang, Zh./Kong L., A Dense Stereo Matching Algorithm based on Triangulation, Journal of Computational Information Systems 8(1), 283-292. 